\title{
Du gros orteil sur la table basse
}

\section{Eberhard Wolff}

Prof. Dr rer. soc., rédacteur culture, histoire, société

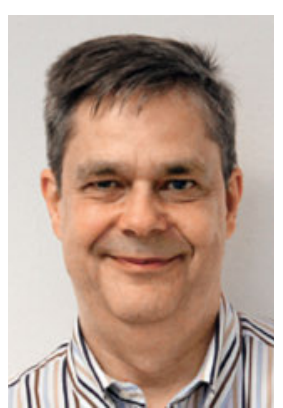

Longtemps, il m'a semblé que c'était l'une des situations les plus étranges de toute ma vie. Il y a bien des années, je me suis retrouvé dans le salon bourgeois de la veuve âgée, entre-temps décédée, d'un médecin. Sur la table basse était posé un bocal contenant un gros orteil humain. Apparemment, celui-ci avait été amputé à un patient diabétique longtemps auparavant et préparé pour être conservé. "Creepy» diraient les jeunes aujourd'hui.

Le gros orteil n'avait pas été posé sur la table basse par mégarde, mais délibérément. Peut-être même avait-il été mis là pour moi, l'historien de la médecine en visite.

Si je me souviens bien, aux yeux de la veuve, cette préparation incarnait l'efficacité du traitement du diabète que son mari avait réalisé de son vivant. Parce que le patient avait été soigné grâce à cette méthode, il n'avait dû être amputé que de l'orteil et non de tout le pied ou même de la jambe.

Changement de décor. Sur son dernier disque, l'auteurcompositeur-interprète suédois Jens Lekman publie le titre un peu tordu "Evening Prayer», qui parle de son ami Babak. Babak était encore à l'école lorsqu'il a fallu lui retirer une tumeur dans le dos. De toute évidence, on lui a ensuite remis l'excroissance pour qu'il la ramène chez lui. Babak s'est en effet servi de l'imprimante 3D de l'école pour en créer une réplique en plastique gris. Là encore, c'est plutôt creepy.

Dans la chanson, lorsque les deux amis vont boire une bière, Babak emporte la reproduction et la pose sur la table en disant: "So, this is what caused all my fears". La petite maquette de tumeur grise rend ses angoisses palpables. A la fin, la barmaid voit l'objet, trouve qu'il a quelque chose de cool et se le fait offrir par Babak.

Que font les gens des parties de leur corps qui se détachent ou sont éliminées par ablation? De même, que font-ils des corps des défunts? La plupart du temps, ces (parties du) corps disparaissent plus ou moins discrètement, que ce soit à l'hôpital, dans un cercueil, un crématorium, au cabinet du dentiste ou chez le coiffeur. Mais certaines personnes conservent ces vestiges de la vie. S'agit-il de creeps, à l'image de Norman Bates, qui continuait de prendre soin du cadavre momifié de sa mère au sous-sol dans le film de Hitchcock «Psychose»? Conserver les morts ou du moins des parties d'eux est plus fréquent qu'on le pense au premier abord. Il suffit de songer à l'Eglise et à ses reliques de saints d'une macabre beauté. Mais les cheveux de morts très ordinaires ont également été transformés en tableaux ou portés dans des broches.

Une usine de pressage britannique fabrique depuis des années des vinyles enrichis des cendres de défunts. Le cher disparu grésille alors à sa manière à la lecture du disque. D'autres font presser les cendres d'êtres chers en diamants.

La conservation de certaines parties du corps n'est pas non plus si anormale. De très nombreuses personnes gardent chez elles les vis et plaques d'un traitement d'ostéosynthèse. Pendant des semaines ou des mois, ces pièces sont restées en elles, sont devenues des parties d'elles. Elles évoquent désormais un accident, une maladie et, espérons-le, une guérison.

La rédaction de ces lignes fait remonter en moi le souvenir de la maison de mes parents. Sur le buffet du salon trônait un coffret en bois finement sculpté. A l'origine, mon grand-père y conservait ses cigares. Par la suite, on y rangea également des boucles de cheveux et des dents de lait des enfants. Les reliques d'une famille de la petite bourgeoisie.

Les anciennes parties d'un corps (en particulier du sien) ne sont pas seulement de la matière morte. Lorsqu'elles sont conservées, elles acquièrent une vie propre. Elles deviennent porteuses de sens. Elles représentent l'individu, sa personnalité, des périodes de sa vie. Elles symbolisent des événements, des idées, des sentiments. Ou inversement, elles représentent les idées que nous projetons sur elles.

Et voilà que je viens d'apprendre que ma propre grandmère conservait deux orteils amputés bien en évidence dans le buffet de son salon. Vue sous cet angle, la situation la plus étrange de toute ma vie n'a rien de bien exceptionnel. Il n'empêche que tout cela est un peu creepy. 